\title{
SOLUTIONS OF A NONHYPERBOLIC PAIR OF BALANCE LAWS
}

\author{
Michael SEver ${ }^{1}$
}

\begin{abstract}
We describe a constructive algorithm for obtaining smooth solutions of a nonlinear, nonhyperbolic pair of balance laws modeling incompressible two-phase flow in one space dimension and time. Solutions are found as stationary solutions of a related hyperbolic system, based on the introduction of an artificial time variable. As may be expected for such nonhyperbolic systems, in general the solutions obtained do not satisfy both components of the given initial data. This deficiency may be overcome, however, by introducing an alternative "solution" satisfying both components of the initial data and an approximate form of a corresponding linearized system.
\end{abstract}

Mathematics Subject Classification. 35M99, 35Q35, 76T10.

Received: April 14, 2004.

\section{INTRODUCTION}

We consider smooth solutions of a pair of balance laws of the form

$$
\begin{gathered}
\beta_{t}+(K \beta-v b(\beta))_{x}=0, \\
v_{t}+\left(K v-\frac{1}{2} v^{2} b_{\beta}(\beta)\right)_{x}=F(x, v, \beta), \quad 0<x<L, \quad t>0,
\end{gathered}
$$

with

$$
b(\beta)=(\beta-1)(1+\lambda-\beta) / \beta,
$$

with dependent variables assuming values

$$
1 \leq \beta(x, t) \leq 1+\lambda, \quad v(x, t) \in \mathbb{R} .
$$

In (1.1)-(1.3), $\lambda>0, \quad K \in \mathbb{R}, \quad F:(0, L) \times \mathbb{R} \times\{1,1+\lambda] \rightarrow \mathbb{R}$ are given.

This system arises as a model of incompressible two-phase flow in one space dimension and time, making the single-pressure approximation $[5,8]$. The variable $\beta$ is a linear measure of the local volume fraction, with $\beta=1$ corresponding to the heavier fluid, of density $1+\lambda$, and $\beta=1+\lambda$ corresponding to the lighter fluid of unit density. Indeed, equation (1.1) even in weak form is an expression of conservation of mass of each separate phase. As against this, the variable $v$ is a weighted velocity variable, not necessarily a conserved (or balanced)

Keywords and phrases. Nonhyperbolic balance laws, incompressible two-fluid flow.

1 Department of Mathematics, The Hebrew University, Jerusalem, Israel. server@math.huji.ac.il 
quantity in the context of discontinuous solutions. The function $F$ describes the applied forces; for example, for $x$ increasing vertically and $F$ including gravity and drag terms, a possible form for $F$ is

$$
F=-g-\sigma v|v|
$$

with $g>0$ the gravitational constant and $\sigma>0$ a drag coefficient [8].

Lack of hyperbolicity is an endemic problem in such models of two-phase flow, particularly in the single pressure approximation $[5,7,12,15]$. For the system $(1.1),(1.2)$, the characteristic speeds are real only in the relatively uninteresting special cases $\beta=1, \beta=1+\lambda$, and $v=0$.

A careful discussion of the mathematical and numerical implications of lack of hyperbolicity in systems of conservation laws has recently been given in [10]. In particular, solutions of the classical initial value problem for (1.1), (1.2), however smooth, must be expected to be absolutely unstable under perturbation. Finding nontrivial solutions as limits of solutions of a regularized problem as the dissipation vanishes is not a realistic approach for this system. A useful discussion of lack of hyperbolicity of systems including (1.1), (1.2) in the context of specific applications is given in [4].

One alternative to regularization of the system (1.1), (1.2) is application of a wave front tracking algorithm [2]. Such an approach in general requires knowledge of the admissible discontinuities for a given system. In the case of the system (1.1), (1.2). discontinuous solutions of this system have been studied $[8,9,13]$.

The system (1.1), (1.2) admits few classical weak solutions, of very limited interest, but does admit singular shocks, solutions valued as measures, obtained as limits of viscous structures. Alternatively, a different derivation of the model of two-phase flow results in a modified form of equation (1.2)[14], for which discontinuous solutions are again obtained as the limits of viscous structures.

The two approaches give the same answer with regard to the set of admissible discontinuities. A discontinuity propagating with speed $s$, connecting $v_{L}, \beta_{L}$ on the left to $v_{R}, \beta_{R}$ on the right is admissible provided that two conditions are satisfied: the Rankine-Hugoniot condition corresponding to (1.1),

$$
(s-K)\left(\beta_{R}-\beta_{L}\right)=-v_{R} b\left(\beta_{R}\right)+v_{L} b\left(\beta_{L}\right),
$$

and an entropy condition, of the form

$$
-v_{R} b_{\beta}\left(\beta_{R}\right) \leq s-K \leq-v_{L} b_{\beta}\left(\beta_{L}\right)
$$

The conditions (1.6), (1.7) supply enough admissible discontinuities to solve the Riemann problem for (1.1), (1.2) (with $F=0$ ), but not for applying wave front tracking to more general Cauchy problems [8]. In particular, in general the solution of "small" Riemann problems is not "small", so continuous but not piecewise constant initial data cannot be handled. Furthermore, the balance term $F$ is problematical, as methods such as that described in [1] depend on hyperbolicity.

Here we discuss an algorithm for approximating nontrivial smooth solutions of the system (1.1), (1.2) based on the well-known technique of introducing an artificial time variable $\tau$ and finding solutions as limits of solutions of a related hyperbolic system as $\tau \rightarrow \infty$. We discuss sufficient conditions that the limit as $\tau \rightarrow \infty$ exists, that the solution so obtained depends continuously on the various problem parameters, and that the solution so obtained is independent of the artificial, "numerical" parameters.

The analytical results presented below are intended to motivate and perhaps justify the computational algorithm, not to delineate its range of applicability. In this spirit we readily acknowledge several important limitations:

First, our present results are "in the small", essentially sufficient conditions under which stationary solutions (in $t$ ) of (1.1), (1.2) are local attractors. These results depend on some qualitative assumptions on the solutions of the related hyperbolic system, expedient but possibly difficult to verify.

Second, our results are limited to special ranges of the problem parameters and conditions on the balance term $F$. In particular, we have no satisfactory way of allowing the boundary conditions at $x=0$ and $x=L$ to depend explicitly on $t$. 
Third, this algorithm finds only smooth solutions. At present we have no way of approximating piecewise smooth solutions, with admissible discontinuities as described above.

Fourth, and perhaps most important, the adopted condition at $t=0$ does not correspond to the expected initial condition for a hyperbolic system of balance laws. Our solutions are uniquely determined by a single linear combination of $v(\cdot, 0)$ and $\beta(\cdot, 0)$. We contend that such is to be expected, in view of the results of [10].

A brief outline of the discussion follows. In Section 2, we discuss the stationary solutions of (1.1), (1.2) and the form of the boundary conditions at $x=0, x=L$. The related hyperbolic system, with $\tau$ as the "timelike" independent variable and $x, t$ as "space-like" variables, is introduced in Section 3 . This system admits a uniformly convex entropy density, and so allows for comparison of smooth solutions with weak solutions satisfying the corresponding entropy inequality [3]. In Section 4, we discuss the particular property of the system (1.1), (1.2) from which the attractor property of the stationary solutions derives. The principal results are then obtained in Section 5. Finally in Section 6 , we show how both $v(\cdot, 0)$ and $\beta(\cdot, 0)$ can be specified by relaxing the requirement of a single-valued solution or by accepting an approximate solution.

\section{Stationary SOLUTiOnS AND BOUNDARY CONDITIONS}

In the context of the application to two-phase flow as described above, we are primarily concerned here with dependent variables

assuming values in $X$,

$$
z=\left(\begin{array}{l}
v \\
\beta
\end{array}\right)
$$

$$
X=\left\{v<0, \quad 1<\beta<(1+\lambda)^{\frac{1}{2}}\right\}
$$

For all $z \in X$, from (1.3),

$$
b(\beta), b_{\beta}(\beta)>0, \quad b_{\beta \beta}(\beta)<0 .
$$

The system (1.1), (1.2) is invariant under scaling in $x, t$, and under scaling in $t, v, K$ (under which $X$ is also invariant). Thus in comparing solutions corresponding to different problem parameters, we shall consider $K$ and $L$ as fixed positive constants.

Boundary conditions at $x=0, \quad x=L$ are conveniently formulated in terms of the normal fluxes, denoted by $q$,

$$
q=\left(\begin{array}{c}
q_{1}(z) \\
q_{2}(z)
\end{array}\right)=\left(\begin{array}{c}
K \beta-v b(\beta) \\
K v-\frac{1}{2} v^{2} b_{\beta}(\beta)
\end{array}\right)
$$

Lemma 2.1. The mapping (2.4) determines an isomorphism of $X$ onto $Y$, given by

$$
Y=\left\{q_{1}>K, q_{2}<0\right\}
$$

Proof. Given $z \in X, q$ is explicitly given in (2.4), and $q \in Y$ follows easily from (2.2)-(2.4). Given $q \in Y$, we solve the second component of (2.4) for negative $v$, using (1.3) and obtaining

$$
-\frac{v}{K}=\frac{\left[1-2 \frac{q_{2}}{K^{2}}\left(\frac{1+\lambda}{\beta^{2}}-1\right)\right]^{\frac{1}{2}}-1}{\left(\frac{1+\lambda}{\beta^{2}}-1\right)}
$$

Using (2.6) in the first component of (2.4), we obtain an equation for $\beta$, again using (1.3),

$$
\left(\frac{\beta}{1+\lambda-\beta}\right)\left(\frac{\frac{q_{1}}{K}-1}{\beta-1}-1\right)-\frac{\left[1-2 \frac{q_{2}}{K^{2}}\left(\frac{1+\lambda}{\beta^{2}}-1\right)\right]^{\frac{1}{2}}-1}{\left(\frac{1+\lambda}{\beta^{2}}-1\right)}=0
$$


Using (2.5) it is readily checked that the left side of $(2.7)$ is monotonically decreasing in the interval $\left(1,(1+\lambda)^{\frac{1}{2}}\right)$, negative for $\beta=(1+\lambda)^{\frac{1}{2}}-0$, and positive for $\beta-1$ sufficiently small. So (2.7) uniquely determines $\beta$ as a function of $q_{1}, q_{2}$, and (2.6) then explicitly determines the corresponding value of $v$.

We use the lemma to parameterize the stationary solutions by the values of $q_{1}, q_{2}$.

Theorem 2.2. Assume $F$ continuous in $x$, locally Lipshitz in $v, \beta$, and satisfying an estimate of the form

$$
|F(x, v, \beta)| \leq c\left(1+v^{2}\right)
$$

then for any $q \in Y$, there is a unique function $\underline{z}$ satisfying

$$
\begin{gathered}
(K \underline{\beta}-\underline{v} b(\underline{\beta}))_{x}=0, \\
\left(K \underline{v}-\frac{1}{2} \underline{v}^{2} b_{\beta}(\underline{\beta})\right)_{x}=F(x, \underline{v}, \underline{\beta}), \quad 0<x<L ; \\
\underline{z}(0) \in X ; \\
K \underline{\beta}(L)-\underline{v}(L) b(\underline{\beta}(L))=q_{1}, \\
K \underline{v}(0)-\frac{1}{2} \underline{v}(0)^{2} b_{\beta}(\underline{\beta}(0))=q_{2} .
\end{gathered}
$$

Remark. In (2.8) and below, c is a generic positive constant.

Proof. From (2.9) and (2.12)

$$
K \underline{\beta}(0)-\underline{v}(0) b(\underline{\beta}(0))=q_{1} .
$$

By appeal to Lemma 2.1, using (2.13), (2.14), we have $\underline{z}(0)$ satisfying (2.11) uniquely determined as a function of $q_{1}, q_{2}$.

Now $(2.9),(2.10)$ are solved for $\underline{v}_{x}, \underline{\beta}_{x}$, obtaining

$$
\begin{gathered}
\underline{v}_{x}=\frac{F}{D(\underline{z})}\left(K-\underline{v} b_{\beta}(\underline{\beta})\right), \\
\underline{\beta}_{x}=\frac{F}{D(\underline{z})} b(\underline{\beta}), \quad 0<x<L,
\end{gathered}
$$

where here and below we use

$$
D(z)=\left(K-v b_{\beta}(\beta)\right)^{2}-\frac{1}{2} v^{2} b(\beta) b_{\beta \beta}(\beta) .
$$

From (1.3), (2.3), there is a positive constant $c$ such that

$$
D(z) \geq c\left(1+v^{2}\right)
$$

for all $v \in \mathbb{R}, 1 \leq \beta \leq 1+\lambda$.

Thus it suffices to show that the initial value problem determined by $(2.15),(2.16)$, with $\underline{z}(0) \in X$ given, has a unique solution, with $\beta(x) \in(1,1+\lambda)$ for all $0 \leq x \leq L$.

Using (2.8) and (2.18), it follows that $|F| / D$ is uniformly bounded. Thus from $(2.15),|v|$ can increase at most exponentially with respect to $x$. From (2.16) and (1.3), $\beta-1$ and $1+\lambda-\beta$ can decrease at most exponentially with $x$. Since $\beta(0) \in(1,1+\lambda), \underline{\beta}(x) \in(1,1+\lambda)$ for all $x \in[0, L]$. The claimed results now follow by assumption on the regularity of $F$.

We next obtain a result on the continuous dependence of stationary solutions on $q_{1}, q_{2}, F$. 
Theorem 2.3. Let $\underline{z}$ be the solution of (2.9)-(2.13) for some given $q_{1}, q_{2}$, and $F$ as in Theorem 2.2. Let $\underline{z}^{\prime}$ be another solution of (2.9)-(2.13), corresponding to values $q_{1}^{\prime}, q_{2}^{\prime}$, and a balance term $F^{\prime}$ of the form

$$
F^{\prime}(x, v, \beta)=F(x, v, \beta)+G(x, v, \beta),
$$

also satisfying the assumptions of Theorem 2.2. Then there is a constant $c_{M}$ depending on

$$
M=\sup _{0 \leq x \leq L}\left(|\underline{v}(x)|+\left|\underline{v}^{\prime}(x)\right|\right)
$$

such that

$$
\sup _{0 \leq x \leq L}\left|\underline{z}(x)-\underline{z}^{\prime}(x)\right|+\sup _{0<x<L}\left|\underline{z}_{x}(x)-\underline{z}_{x}^{\prime}(x)\right| \leq c_{M}\left(\left|q_{1}-q_{1}^{\prime}\right|+\left|q_{2}-q_{2}^{\prime}\right|+\sup _{0<x<L} \mid G\left(x, \underline{v}^{\prime}(x), \underline{\beta}^{\prime}(x) \mid\right)\right.
$$

Proof. Computing the matrix $q_{z}$ from (2.4) and (2.1), using (2.18) we readily obtain

$$
\left|q_{z}^{-1}(z)\right| \leq c
$$

with a constant $c$ independent of $z$. From $(2.13),(2.14)$, using $(2.22)$ we obtain

$$
\left|\underline{z}(0)-\underline{z}^{\prime}(0)\right| \leq c\left(\left|q_{1}-q_{1}^{\prime}\right|+\left|q_{2}-q_{2}^{\prime}\right|\right) ;
$$

the remainder of $(2.21)$ is elementary.

The stationary solutions of primary interest here correspond to moderate values of $q_{1}, q_{2}$, and a balance term $F$ satisfying additional conditions, compatible with the example (1.5).

Theorem 2.4. In addition to the assumptions of Theorem 2.2, assume that $F$ satisfies

$$
F(x, v, \beta) \leq-c<0, \quad 0 \leq x \leq L, \quad-c_{0} \leq v \leq 0, \quad 1 \leq \beta \leq 1+\lambda
$$

for some positive constant $c_{0}$. Then for all sufficiently small $q_{1}-K$ and $\left|q_{2}\right|$, the corresponding stationary solution $\underline{z}$ satisfies

$$
\begin{gathered}
\frac{c\left|q_{2}\right|}{1+\left|q_{2}\right|^{\frac{1}{2}}} \leq-\underline{v}(x) \leq c\left|q_{2}\right|, \\
\underline{\beta}(x)-1 \leq c\left(q_{1}-K+\left|q_{2}\right|\right), \quad 0 \leq x \leq L ; \\
\underline{v}_{x}(x) \leq-c<0, \\
\underline{\beta}_{x}(x)<0, \quad 0<x<L .
\end{gathered}
$$

Proof. From (2.6) and (2.7), $\underline{\beta}(0) \downarrow 1$ and $\underline{v}(0) \uparrow 0$ as $q_{1} \downarrow K$ and $q_{2} \uparrow 0$. Thus using (2.22), it follows that (2.26) holds at $x=0$, and then from $(2.6)$ that $(2.25)$ holds at $x=0$. Since $|\underline{v}|$ can increase at most exponentially in $x$, for $\left|q_{2}\right|$ sufficiently small we have $(2.25)$ and

$$
-c_{0} \leq \underline{v}(x)<0
$$

holding for all $0 \leq x \leq L$. From (2.24) and (2.29), $F<0$ throughout the interval $(0, L)$, and $(2.26)-(2.28)$ follow from $(2.15),(2.16)$. 


\section{IMBEDDing IN A HYPERBOLIC SYSTEM}

The system (1.1), (1.2) is of a skew-gradient form [8]

$$
\begin{aligned}
& \beta_{t}+\omega_{v, x}=0 \\
& v_{t}+\omega_{\beta, x}=F
\end{aligned}
$$

with

$$
\omega(z)=K v \beta-\frac{1}{2} v^{2} b(\beta) .
$$

This representation facilitates finding solutions of $(1,1),(1.2)$ as solutions of a related hyperbolic system, stationary with respect to an artificial time variable $\tau$, of the form

$$
\phi_{z, \tau}+\psi_{z, t}+\omega_{z, x}=\left(\begin{array}{c}
0 \\
F
\end{array}\right) .
$$

In $(3.3)$,

$$
\begin{aligned}
\phi(z)=\frac{1}{2}|z|^{2} & =\frac{1}{2}\left(v^{2}+\beta^{2}\right) \\
\psi(z) & =v \beta
\end{aligned}
$$

as $\phi$ is strictly convex in $z$, the system $(3.3)$ is hyperbolic $[6,11]$ with $\tau$ as the time-like independent variable.

We consider solutions of (3.3) in the region

$$
(x, t, \tau) \in \Omega=(0, L) \times(0, \infty) \times(0, \infty)
$$

consideration of the characteristic speeds for the system (3.3) determines that a single boundary condition is needed on each of the boundary segments $x=0, x=L, t=0$, and a limit condition at $t=\infty$.

For some parameters $\nu, \eta \in(0,1)$, we take boundary conditions at $t=0$ and $t=\infty$ of the form

$$
\begin{gathered}
\nu \beta(x, 0)+(1-\nu) v(x, 0)=f(x), \quad 0<x<L \\
\eta(\beta(x, \infty)-\underline{\beta}(x))-(1-\eta)(v(x, \infty)-\underline{v}(x))=0, \quad 0<x<L .
\end{gathered}
$$

Motivation for boundary conditions of this form will be given in Section 4 .

The data at $t=0$ is limited to the value of $\nu$ and the values of the scalar function $f$, assumed of class $C^{1}$ in the interval $(0, L)$.

In $(3.8), \beta(\cdot, \infty), v(\cdot, \infty)$ are understood as limiting values as $t \rightarrow \infty$, and we regard the stationary solution $\underline{z}$ as uniquely determined from the values of $q_{1}$ and $q_{2}$, by appeal to Theorem 2.2 .

Throughout we shall apply boundary conditions at $x=0, x=L$, of the same form as $(2.12),(2.13)$,

$$
\begin{gathered}
K \beta(L, t, \tau)-v(L, t, \tau) b(\beta(L, t, \tau))=q_{1}, \quad t, \tau \geq 0, \\
K v(0, t, \tau)-\frac{1}{2} v(0, t, \tau)^{2} b_{\beta}(\beta(0, t, \tau))=q_{2}, \quad t, \tau \geq 0 .
\end{gathered}
$$

The "initial data" at $\tau=0$ shall be assumed close to the corresponding stationary solution, smooth and satisfying (3.7), (3.8), (3.9), and (3.10), and with

$$
\int_{0}^{\infty} \int_{0}^{L} \mathrm{e}^{c_{1} t}|z(x, t, 0)-\underline{z}(x)|^{2} \mathrm{~d} x \mathrm{~d} t=c,
$$

for some positive constant $c_{1}$. 
With this initial/boundary data, we assume the existence of an entropy weak solution of (3.3), at least until such $\tau$ as it becomes infinite (in $L_{2}$ ). Uniqueness is not needed; an entropy inequality of the form $[L]$

$$
\phi_{\tau}+\psi_{t}+\zeta_{x}-\beta F \leq 0
$$

suffices. In general, (3.12) is understood in the sense of distributions; smooth solutions of (3.3) satisfy (3.12) strongly and with equality. In (3.12) and hereafter $\zeta$ denotes the Lagrange transform of $\omega$, obtained from $(3.2),(1.3)$,

$$
\begin{aligned}
\zeta(z) & =z \omega_{z}(z)-\omega(z) \\
& =K v \beta-v^{2}\left(1+\frac{\lambda}{2}-\beta\right) .
\end{aligned}
$$

Additional qualitative assumptions are needed on the regularity of entropy weak solutions of (3.3). Specifically, we assume that for any $\varepsilon>0$ there exists $\delta>0, c_{2}>0, c_{3}$ such that if

$$
\begin{aligned}
& \sup _{\substack{0<x<L \\
t>0}}\left(|z(x, t, 0)-\underline{z}(x)|+\left|z_{x}(x, t, 0)-\underline{z}_{x}(x)\right|+\left|z_{t}(x, t, 0)\right|\right), \\
& \sup _{0<\tau^{\prime}<\tau}\left(\int_{0}^{\infty} \int_{0}^{L} \mid z\left(x, t, \tau^{\prime}|-\underline{z}(x)|^{2} \mathrm{~d} x \mathrm{~d} t\right)^{\frac{1}{2}} \leq \delta,\right.
\end{aligned}
$$

then

$$
\sup _{\substack{0<x<L \\ t>0}}|z(x, t, \tau)-\underline{z}(x)|, \sup _{\substack{0<x<L \\ t<c_{2} \tau-c_{3}}}\left|z_{x}(x, t, \tau)-\underline{z}_{x}(x)\right| \leq \varepsilon .
$$

In (3.14), (3.15), $\delta$ may depend on $q_{1}, q_{2}$ but not on $\nu, f$.

The first condition in (3.15) involves an assumption on the data at $\tau=0$, namely that this is chosen to avoid focusing in the sense of the linear wave equation in several space variables. We shall show in Section 5 , however, that our results are otherwise independent of the artificial initial data.

The constants $c_{2}, c_{3}$ in the second condition in (3.15) may depend on the particular solution $z$. This condition is rationalized on the grounds that for the system (3.3), there are no planar shocks connecting two states in $X$ with speeds close to zero. Thus while shocks are expected to form spontaneously for the system (3.3), they are expected to leave the bounded subsets of $(0, L) \times(0, \infty)$ via the boundaries or else by movement to large values of $t$ as $\tau$ increases.

\section{A COMPARISON THEOREM}

Our principal results are obtained by comparison of two solutions of (3.3): a smooth solution $z$, corresponding to parameters $q_{1}, q_{2}, \nu, f, \eta, F$; and an entropy weak solution $z^{\prime}$, corresponding to parameters $q_{1}^{\prime}, q_{2}^{\prime}, \nu^{\prime}, f^{\prime}, \eta^{\prime}, F^{\prime}$. Throughout we use $F^{\prime}$ of the form (2.19), for some function $G$.

In comparing the solution values, we use notation of the form

$$
\begin{gathered}
{[z]=z-z^{\prime},} \\
Q(\omega)=\omega(t)-\omega\left(z^{\prime}\right)-\omega_{z}\left(z^{\prime}\right)[z], \\
Q^{*}\left(\omega_{z}\right)=\omega_{z}\left(z^{\prime}\right)-\omega_{z}(z)+\omega_{z z}(z)[z],
\end{gathered}
$$

extended in the obvious manner to other potential functions $\phi, \psi$. Inner products and matrix-vector products in $l_{2}$ are understood in (4.2), (4.3) and below. 
The potential functions $\phi$ and $\psi$ are homogeneous of degree two in $z$; their quadratic parts are of simple form

$$
Q(\phi)=\frac{1}{2}[z]^{2} ; \quad Q(\psi)=[v][\beta] ; \quad Q^{*}\left(\phi_{z}\right)=Q^{*}\left(\psi_{z}\right)=0 .
$$

We use here a variation of a standard estimate [3] used to prove uniqueness of smooth solutions of systems of conservation laws equipped with convex entropies. The argument is somewhat simplified in the symmetric coordinates $z$.

Lemma 4.1. Given $w \in C^{1}\left[y_{0}, y_{1}\right] \rightarrow X, w^{\prime} \in B V\left[y_{0}, y_{1}\right] \rightarrow X, \quad \Theta \in C^{1}\left[y_{0}, y_{1}\right] \rightarrow \mathbb{R}_{+}$and $\chi \in C^{2}: X \rightarrow \mathbb{R}$, we have

$$
\begin{aligned}
& \int_{y_{0}}^{y_{1}} \Theta(y)\left[\left(w \chi_{w}(w)-\chi(w)\right)_{y}-w^{\prime} \chi_{w, y}(w)-w \chi_{w, y}\left(w^{\prime}\right)+\left(w^{\prime} \chi_{w}\left(w^{\prime}\right)-\chi\left(w^{\prime}\right)\right)_{y}\right] \mathrm{d} y \\
&=\left.(Q(\chi) \Theta)\right|_{y=y_{0}} ^{y_{1}}-\int_{y_{0}}^{y_{1}} \Theta_{y} Q(\chi) \mathrm{d} y+\int_{y_{0}}^{y_{1}} \Theta w_{y} Q^{*}\left(\chi_{w}\right) \mathrm{d} y .
\end{aligned}
$$

Proof. Partial integrations give the left side of (4.5) equal to

$$
\begin{aligned}
& \left.\Theta\left[w \chi_{w}(w)-\chi(w)+w^{\prime} \chi_{w}\left(w^{\prime}\right)-\chi\left(w^{\prime}\right)-w \chi_{w}\left(w^{\prime}\right)\right]\right|_{y=y_{0}} ^{y_{1}} \\
& \quad+\int_{y_{0}}^{y_{1}} \Theta_{y}\left[-w \chi_{w}(w)+\chi(w)-w^{\prime} \chi_{w}\left(w^{\prime}\right)+\chi\left(w^{\prime}\right)+w \chi_{w}\left(w^{\prime}\right)\right] \mathrm{d} y+\int_{y_{0}}^{y_{1}} \Theta w_{y}\left[-\chi_{w w}(w) w^{\prime}+\chi_{w}\left(w^{\prime}\right)\right] \mathrm{d} y .
\end{aligned}
$$

Using the identity

$$
\left.\Theta\left(2 \chi(w)-\chi_{w}(w) w\right)\right|_{y_{0}} ^{y_{1}}=\int_{y=y_{0}}^{y_{1}} \Theta_{y}\left(2 \chi(w)-w \chi_{w}(w)\right) \mathrm{d} y+\int_{y_{0}}^{y_{1}} \Theta\left[\chi_{w}(w)-w \chi_{w w}(w)\right] w_{y} \mathrm{~d} y
$$

and (4.2), (4.3), the expression (4.6) is equal to the right side of (4.5).

Lemma 4.2. Assume $z, z^{\prime}$ each an entropy weak solution of (3.3), $\theta=\theta(x) \in C^{1}[0, L] \rightarrow \mathbb{R}_{+}$and $\hat{\theta}=\hat{\theta}(t, \tau) \in$ $W^{1, \infty}: \mathbb{R}_{+} \times \mathbb{R}_{+} \rightarrow \mathbb{R}_{+}$. Assume in addition that $\left|z_{x}\right|$ is uniformly bounded on the region $x \in(0, L),(t, \tau)$ in the support of $\hat{\theta}$.

Then for all $0 \leq \tau_{0}<\tau_{1}$,

$$
\begin{aligned}
&\left.\frac{1}{2} \int_{0}^{\infty} \int_{0}^{L} \theta \hat{\theta}[z]^{2} \mathrm{~d} x \mathrm{~d} t\right|_{\tau=\tau_{0}} ^{\tau_{1}}+\left.\int_{\tau_{0}}^{\tau_{1}} \int_{0}^{L} \theta \hat{\theta}[v][\beta]\right|_{t=0} ^{\infty}+\int_{\tau_{0}}^{\tau_{1}} \int_{0}^{\infty} \hat{\theta}\left(\left.\theta Q(\omega)\right|_{x=0} ^{L}\right) \mathrm{d} t \mathrm{~d} \tau \\
&-\int_{\tau_{0}}^{\tau_{1}} \int_{0}^{\infty} \int_{0}^{L} \theta\left(\frac{1}{2}[z]^{2} \hat{\theta}_{\tau}+[v][\beta] \hat{\theta}_{t}\right) \mathrm{d} x \mathrm{~d} t \mathrm{~d} \tau+\int_{\tau_{0}}^{\tau_{1}} \int_{0}^{\infty} \int_{0}^{L} \hat{\theta} A \mathrm{~d} x \mathrm{~d} t \mathrm{~d} \tau \leq 0,
\end{aligned}
$$

with the abbreviation

$$
A=-Q(\omega) \theta_{x}+\theta z_{x} Q^{*}\left(\omega_{z}\right)-\theta[\beta][F] .
$$


Proof. From (3.3) and the entropy inequality (3.12),

$$
\begin{aligned}
0 \geq & \int_{\tau_{0}}^{\tau_{1}} \int_{0}^{\infty} \int_{0}^{L} \theta \hat{\theta}\left[\phi_{\tau}(z)+\psi_{t}(z)+\zeta_{x}(z)-\beta F-z^{\prime}\left(\phi_{z, \tau}(z)+\psi_{z, t}(z)+\omega_{z, x}(z)-\left(\begin{array}{c}
0 \\
F
\end{array}\right)\right)\right. \\
& \left.-z\left(\phi_{z, \tau}\left(z^{\prime}\right)+\psi_{z, t}\left(z^{\prime}\right)+\omega_{z, x}\left(z^{\prime}\right)-\left(\begin{array}{c}
0 \\
F^{\prime}
\end{array}\right)\right)+\phi_{\tau}\left(z^{\prime}\right)+\psi_{t}\left(z^{\prime}\right)+\zeta_{x}\left(z^{\prime}\right)-\beta^{\prime} F^{\prime}\right] \mathrm{d} x \mathrm{~d} t \mathrm{~d} \tau \\
= & \int_{0}^{\infty} \int_{0}^{L} \theta \int_{\tau_{0}}^{\tau_{1}} \hat{\theta}\left(\phi_{\tau}(t)-z^{\prime} \phi_{z, \tau}(z)-z \phi_{z, \tau}\left(z^{\prime}\right)+\phi_{\tau}\left(z^{\prime}\right)\right) \mathrm{d} \tau \mathrm{d} x \mathrm{~d} t \\
& +\int_{\tau_{0}}^{\tau_{1}} \int_{0}^{L} \theta \int_{0}^{\infty} \hat{\theta}\left(\psi_{t}(z)-z^{\prime} \psi_{z, t}(z)-z \psi_{z, t}\left(z^{\prime}\right)+\psi_{t}\left(z^{\prime}\right)\right) \mathrm{d} t \mathrm{~d} x \mathrm{~d} \tau \\
& +\int_{\tau_{0}}^{\tau_{1}} \int_{0}^{\infty} \hat{\theta} \int_{0}^{L} \theta\left(\zeta_{x}(z)-z^{\prime} \omega_{z, x}(z)-z \omega_{z, x}\left(z^{\prime}\right)+\zeta_{x}\left(z^{\prime}\right)\right) \mathrm{d} x \mathrm{~d} t \mathrm{~d} \tau \\
& -\int_{\tau_{0}}^{\tau_{1}} \int_{0}^{\infty} \int_{0}^{L} \theta \hat{\theta}[\beta][F] \mathrm{d} x \mathrm{~d} t \mathrm{~d} \tau .
\end{aligned}
$$

Lemma 4.1 is applied in each of the first three right-hand terms of (4.10), with $y=\tau, t, x, \chi=\phi, \psi, \omega$, and $\Theta=\hat{\theta}(t, \cdot), \hat{\theta}(\cdot, \tau), \theta$, respectively. The functions and $\phi$ and $\psi$, being homogeneous of degree two in $z$, are their own Lagrange transforms; in the third application of Lemma 4.1 we use (3.13). Collecting the results and using (4.4) gives (4.8), (4.9).

Upper bounds for the first term in (4.8) are obtained from lower bounds on the remaining terms. For the second and third terms, such are obtained from the explicit form of the boundary conditions (3.9), (3.10), (3.7), and (3.8) satisfied by each of $z$ and z'. The fourth term in (4.8) is exploited by the choice of $\hat{\theta}$.

The present results depend on special properties of the system (1.1), (1.2), as reflected in an estimate for the last term in (4.8).

Lemma 4.3. Assume a balance term $F$ of class $C^{1}$ in a region $0<x<L, \quad-c_{0}<v<0,1<\beta<(1+\lambda)^{1 / 2}$, satisfying (2.8), (2.24), and

$$
F_{v}(x, 0-, 1+), F_{\beta}(x, 0-, 1+) \leq 0,0<x<L .
$$

Assume values of $K, \lambda$ such that

$$
K\left|F_{v}(x, 0-, 1+)\right|<\lambda|F(x, 0,1)|, \quad 0<x<L .
$$

Let $\underline{z}$ be the stationary solution corresponding to $q_{1}, q_{2}, F$, and determine $\theta$ from

$$
\left(\frac{\underline{v} b_{\beta}(\underline{\beta})}{\theta}\right)_{x}+\frac{F_{v}(\cdot, \underline{v}, \underline{\beta})}{\theta}=0, \quad 0<x<L ; \quad \theta(0)=1 .
$$

Then for $q_{1}, q_{2} \in Y$ (determined from (2.5)) with $q_{1}-K$ and $\left|q_{2}\right|$ nonzero and sufficiently small, there exists a constant $\gamma>0$ such that

$$
A \geq \theta[z]^{2}\left(2 \gamma-O\left(|z-\underline{z}|+\left|z^{\prime}-\underline{z}\right|+\left|z_{x}-\underline{z}_{x}\right|\right)-\frac{\theta}{\gamma} G\left(\cdot, v^{\prime}, \beta^{\prime}\right)^{2} .\right.
$$


Remark. The constant $\gamma$, and indeed all constants $c$ in this proof, depend on the values of $q_{1}, q_{2}$, and on $F$. Proof. From (3.2), (4.2) and (4.3),

$$
\begin{aligned}
Q(\omega)= & -\frac{1}{2} b(\beta)[v]^{2}-v^{\prime} b_{\beta}\left(\beta^{\prime}\right)[v][\beta]-v^{\prime}\left(v-\frac{1}{2} v^{\prime}\right)\left(b(\beta)-b\left(\beta^{\prime}\right)-b_{\beta}\left(\beta^{\prime}\right)[\beta]\right) \\
= & -\frac{1}{2} b(\underline{\beta})[v]^{2}-\underline{v} b_{\beta}(\underline{\beta})[v][\beta]-\frac{1}{4} \underline{v}^{2} b_{\beta \beta}(\underline{\beta})[\beta]^{2} \\
& +O\left([z]^{2}\left(|z-\underline{z}|+\left|z^{\prime}-\underline{z}\right|\right),\right.
\end{aligned}
$$

and

$$
\begin{aligned}
Q^{*}\left(\omega_{z}\right)= & \left(\begin{array}{l}
-b_{\beta}(\beta)[v][\beta]-v^{\prime}\left(b\left(\beta^{\prime}\right)-b(\beta)+b_{\beta}(\beta)[\beta]\right) \\
-\frac{1}{2} b_{\beta}\left(\beta^{\prime}\right)[v]^{2}-v b_{\beta \beta}(\beta)[v][\beta]-v\left(v^{\prime}-\frac{1}{2} v\right)\left(b_{\beta}\left(\beta^{\prime}\right)-b_{\beta}(\beta)+b_{\beta \beta}(\beta)[\beta]\right)
\end{array}\right) \\
= & \left(\begin{array}{l}
-b_{\beta}(\underline{\beta})[v][\beta]-\frac{1}{2} \underline{v}\left(b_{\beta \beta}(\underline{\beta})[\beta]^{2}\right. \\
-\frac{1}{2} b_{\beta}(\underline{\beta})[v]^{2}-\underline{v} b_{\beta \beta}(\underline{\beta})[v][\beta]-\underline{v}^{2} b_{\beta \beta \beta}(\underline{\beta})[\beta]^{2}
\end{array}\right) \\
& +O\left([z]^{2}\left(|z-\underline{z}|+\left|z^{\prime}-\underline{z}\right|\right) .\right.
\end{aligned}
$$

Using (4.15), (4.16) in (4.9), we obtain

$$
\begin{aligned}
A= & \frac{1}{2}\left(b(\underline{\beta}) \theta_{x}-\theta \beta_{x} b_{\beta}(\underline{\beta})\right)[v]^{2} \\
& +\left[\theta_{x} \underline{v} b_{\beta}(\underline{\beta})-\theta v_{x} b_{\beta}(\underline{\beta})-\theta \beta_{x} \underline{v} b_{\beta \beta}(\underline{\beta})-\theta F_{v}(\cdot, v, \beta)\right][v][\beta] \\
& +\left[\frac{1}{4} \theta_{x} \underline{v}^{2} b_{\beta \beta}(\underline{\beta})-\frac{1}{2} \theta v_{x} \underline{v} b_{\beta \beta}(\underline{\beta})-\frac{1}{4} \theta \beta_{x} \underline{v}^{2} b_{\beta \beta \beta}(\underline{\beta})-\theta F_{\beta}(\cdot, v, \beta)\right][\beta]^{2} \\
& +\theta[\beta]\left[F\left(\cdot, v^{\prime}, \beta^{\prime}\right)-F(\cdot, v, \beta)+F_{v}(\cdot, v, \beta)[v]+F_{\beta}(\cdot, v, \beta)[\beta]\right] \\
& +\theta[\beta] G\left(\cdot, v^{\prime}, \beta^{\prime}\right)+O\left([z]^{2}\left(|z-\underline{z}|+\left|z^{\prime}-\underline{z}\right|\right)\right) \\
= & -\frac{1}{2} \theta^{2}\left(\frac{b(\underline{\beta})}{\theta}\right)_{x}[v]^{2}-\theta\left[\theta\left(\frac{\underline{v} b_{\beta}(\underline{\beta})}{\theta}\right)_{x}+F_{v}(\cdot, \underline{v}, \underline{\beta})\right][v][\beta] \\
& -\theta\left[\frac{1}{4} \theta\left(\frac{\underline{v}^{2} b_{\beta \beta}(\underline{\beta})}{\theta}\right)_{x}+F_{\beta}(\cdot, \underline{v}, \underline{\beta})\right][\beta]^{2}+\theta[\beta] G\left(\cdot, v^{\prime}, \beta^{\prime}\right) \\
& +O\left([z]^{2}\left(|z-\underline{z}|+\left|z^{\prime}-\underline{z}\right|+\left|z_{x}-\underline{z}_{x}\right|\right)\right) .
\end{aligned}
$$

For $q_{1}, q_{2}$ satisfying (2.5) with $q_{1}-K$ and $q_{2}$ sufficiently small, from (2.25), (2.26) we have $\underline{\beta}(x)-1$ and $|v(x)|$ as small as we wish. Then using (4.11) and the regularity assumption on $F$, we have

$$
F_{v}(x, \underline{v}(x), \underline{\beta}(x)), \quad F_{\beta}(x, \underline{v}(x), \underline{\beta}(x)) \leq \alpha, \quad 0<x<L,
$$

where $\alpha$ can be made arbitrarily small by the choice of $q_{1}$ and $q_{2}$. 
Rewriting (4.13) in the form

$$
-\frac{\underline{v} b_{\beta}(\underline{\beta}) \theta_{x}}{\theta}=-b_{\beta}(\underline{\beta}) \underline{v}_{x}-\underline{v} b_{\beta \beta}(\underline{\beta}) \underline{\beta}_{x}-F_{v}(\cdot, \underline{v}, \underline{\beta})
$$

using $(2.3),(2.27),(2.28)$, and (4.18) in (4.19), we obtain

$$
\theta_{x} \geq c>0
$$

Then from (2.28), (2.3) and (4.20)

$$
\left(\frac{b(\underline{\beta})}{\theta}\right)_{x} \leq-c<0
$$

Using (4.13), (2.15), (2.16), (1.3) and the assumption (4.12), with $\underline{\beta}-1$ and $|v|$ sufficiently small we have

$$
\begin{aligned}
\left(\frac{\underline{v}^{2} b_{\beta \beta}(\underline{\beta})}{\theta}\right)_{x}< & \frac{\underline{v}}{\theta}\left[b_{\beta \beta}(\underline{\beta}) \frac{F(\cdot, \underline{v}, \underline{\beta})}{D(\underline{z})}\left(K-\underline{v} b_{\beta}(\underline{\beta})\right)\right. \\
& \left.-\frac{b_{\beta \beta}(\underline{\beta})}{b_{\beta}(\underline{\beta})} F_{v}(\cdot, \underline{v}, \underline{\beta})-\frac{\underline{v} b(\underline{\beta}) b_{\beta}(\underline{\beta})}{D(\underline{z})} F(\cdot, \underline{v}, \underline{\beta})\right] \\
\leq & -c<0 .
\end{aligned}
$$

Using (4.13), (4.18), (4.21) and (4.22) in (4.17) now gives (4.14).

We continue with two lemmas giving lower bounds for the "boundary terms" in (4.8).

Lemma 4.4. Assume $z$ and $z^{\prime}$ each satisfying boundary conditions of the form (3.9), (3.10), $\theta(0), \theta(L)>0$ and $\hat{\theta}$ nonnegative. Then

$$
\int_{0}^{\infty} \hat{\theta}(t, \tau)\left(\left.\theta Q(\omega)\right|_{x=0} ^{L}\right) \mathrm{d} t \geq-c\left(\left|q_{1}-q_{1}^{\prime}\right|^{2}+\left|q_{2}-q_{2}^{\prime}\right|^{2}\right) \int_{0}^{\infty} \hat{\theta}(t, \tau) \mathrm{d} t .
$$

Proof. It will suffice to show that

$$
\begin{aligned}
& Q(\omega) \geq-c\left(\left|q_{1}-q_{1}^{\prime}\right|^{2}+\left|q_{2}-q_{2}^{\prime}\right|^{2}\right) \text { at } x=L ; \\
& Q(\omega) \leq c\left(\left|q_{1}-q_{1}^{\prime}\right|^{2}+\left|q_{2}-q_{2}^{\prime}\right|^{2}\right) \quad \text { at } x=0 .
\end{aligned}
$$

For $z^{\prime \prime} \in X$, let

$$
q\left(z^{\prime \prime}\right)=\omega_{z}\left(z^{\prime \prime}\right)
$$

as given in (2.4). Observing that

$$
\operatorname{det} \omega_{z z}\left(z^{\prime \prime}\right)=-D\left(z^{\prime \prime}\right)<0
$$

from $(2.17),(2.18)$, it follows that $q\left(z^{\prime \prime}\right)$ are local coordinates for phase space, and that [11]

$$
z^{\prime \prime}=\zeta_{q}\left(q\left(z^{\prime \prime}\right)\right)
$$

with $\zeta$ the Lagrange transform of $\omega$ as given in (3.11), regarded as a function of $q$. 
From (4.2), using (3.13), (4.25) and (4.27),

$$
\begin{aligned}
Q(\omega) & =\omega(z)-\omega\left(z^{\prime}\right)-\omega_{z}\left(z^{\prime}\right)[z] \\
& =\zeta\left(q^{\prime}\right)-\zeta(q)+\zeta_{q}(q)[q] \\
& =\frac{1}{2}[q]^{\dagger} \zeta_{q q}(q)[q]+O\left([q]^{3}\right) .
\end{aligned}
$$

At $x=L$, from (3.9) we specify $q_{1}=q_{1}(z(L)), q_{1}^{\prime}=q_{1}\left(z^{\prime}(L)\right)$, and (4.28) becomes

$$
Q(\omega) \geq \frac{1}{2}\left[q_{2}\right]^{2} \zeta_{q_{2} q_{2}}-c\left[q_{1}\right]^{2}-c\left|\left[q_{1}\right]\left[q_{2}\right]\right|-O\left([q]^{3}\right) .
$$

At $x=0$, from (3.10) we specify $q_{2}=q_{2}(z(0)), q_{2}^{\prime}=q_{2}\left(z^{\prime}(0)\right)$, and (4.28) becomes

$$
Q(\omega) \leq \frac{1}{2}\left[q_{1}\right]^{2} \zeta_{q_{1} q_{1}}+c\left[q_{2}\right]^{2}+c\left|\left[q_{1}\right]\left[q_{2}\right]\right|+O\left([q]^{3}\right) .
$$

Thus from (4.29), (4.30), to obtain (4.24) it will suffice to show

$$
\zeta_{q_{1} q_{1}}(q)<0<\zeta_{q_{2} q_{2}}(q)
$$

Using (4.27), (2.4), (2.3), (2.17), we have

$$
\begin{aligned}
\zeta_{q_{1} q_{1}} & =v_{q_{1}} \\
& =1 /\left.\frac{\partial q_{1}}{\partial v}\right|_{q_{2} \text { constant }} \\
& =\frac{v^{2}}{2} b_{\beta \beta}(\beta) / D(z) \\
& <0, \\
\text { and } \quad \zeta_{q_{2} q_{2}} & =\beta_{q_{2}} \\
& =1 /\left.\frac{\partial q_{2}}{\partial \beta}\right|_{q_{1} \text { constant }} \\
& =b(\beta) / D(z) \\
& >0 .
\end{aligned}
$$

Lemma 4.5. Assume $\theta$ positive and bounded, $\hat{\theta}$ nonnegative, $z$ satisfying (3.7), (3.8) and $z^{\prime}$ satisfying similar conditions of the form

$$
\begin{gathered}
\nu^{\prime} \beta^{\prime}(x, 0)+\left(1-\nu^{\prime}\right) v^{\prime}(x, 0)=f^{\prime}(x), \quad 0<x<L \\
\eta^{\prime}\left(\beta^{\prime}(x, \infty)-\underline{\beta}^{\prime}(x)\right)-\left(1-\eta^{\prime}\right)\left(v^{\prime}(x, \infty)-\underline{v}^{\prime}(x)\right)=0, \quad 0<x<L .
\end{gathered}
$$

Then

$$
\begin{aligned}
\left.\int_{0}^{L} \theta \hat{\theta}[v][\beta] \mathrm{d} x\right|_{t=0} ^{\infty} \geq-\frac{c \hat{\theta}(0, \tau)}{\nu(1-\nu)}\left([\nu]^{2}+\int_{0}^{L}\right. & \left.\left(f(x)-f^{\prime}(x)\right)^{2} \mathrm{~d} x\right) \\
& -\frac{c \hat{\theta}(\infty, \tau)}{\eta(1-\eta)}\left([\eta]^{2}+\left[q_{1}\right]^{2}+\left[q_{2}\right]^{2}+\sup _{0<x<L} G\left(x, \underline{v}^{\prime}(x), \underline{\beta}^{\prime}(x)\right)^{2}\right) .
\end{aligned}
$$


Proof. At $t=0$, from (3.7) and (4.34)

$$
[v][\beta] \leq c \frac{[\nu]^{2}+[f]^{2}}{\nu(1-\nu)}
$$

pointwise in $x$. At $t=\infty$ we have similarly from (3.8) and (4.35)

$$
\begin{aligned}
{[v][\beta] } & \geq-c \frac{[\eta]^{2}+[\underline{z}]^{2}}{\eta(1-\eta)} \\
& \geq \frac{-c}{\eta(1-\eta)}\left([\eta]^{2}+\left[q_{1}\right]^{2}+\left[q_{2}\right]^{2}+\sup _{0<x<L} G\left(x, \underline{v}^{\prime}(x), \underline{\beta}^{\prime}(x)\right)^{2}\right)
\end{aligned}
$$

using theorem 2.3 in the last step. Now (4.36) follows from (4.37), (4.38).

Finally we collect the estimates obtained in Lemmas 4.2, 4.3, 4.4, and 4.5 in (4.8). A Gronwall inequality results, from which we obtain the following comparison theorem. The assumptions of this theorem are simply those of Theorem 2.2 and the above lemmas, restated for clarity; further details of the proof are omitted.

Theorem 4.6. Let $z, z^{\prime}$ be two entropy weak solutions of $(3.3)$ in a region $(0, L) \times(0, \infty) \times\left(\tau_{0}, \tau_{1}\right)$, each satisfying boundary conditions of the form (3.9), (3.10), (3.7), (3.8) (with possibly different parameters).

Assume $q_{1}, q_{2}$, corresponding to $z$, satisfying (2.5) with $q_{1}-K$ and $\left|q_{2}\right|$ sufficiently small.

Assume $\theta$ determined from (4.13) and the corresponding stationary solution $\underline{z}$ obtained from Theorem 2.2.

Assume the initial data for each of $z$ and $z^{\prime}$ satisfies a condition of the form (3.11) with some positive constant $c_{1}$.

Assume F satisfying (2.8), (2.24), (4.11) (4.12) and the regularity assumption of Lemma 4.3.

For a chosen function $\hat{\theta}$, determine a function $\rho$ and a constant $c_{4}$ such that

$$
\hat{\theta}_{\tau}(t, \tau)+\left|\hat{\theta}_{t}(t, \tau)\right| \leq c_{4} \hat{\theta}(t, \tau)+\rho(t, \tau), \quad 0<t<\infty ; \quad \tau_{0}<\tau<\tau_{1}
$$

Finally assume $|z-\underline{z}|,\left|z-z^{\prime}\right|$, and $\left|z_{x}-\underline{z}_{x}\right|$ sufficiently small for $x \in(0, L)(t, \tau)$ in the support of and $\hat{\theta}$ and $\tau_{0} \leq \tau<\tau_{1}$, so that (4.14) holds in the form

$$
A \geq \theta \gamma[z]^{2}-\frac{\theta}{\gamma} G\left(\cdot, v^{\prime}, \beta^{\prime}\right) .
$$

Then assuming that all of the indicated integrals below exist, there is a positive constant $c_{5}$ such that

$$
\begin{aligned}
& \int_{0}^{\infty} \int_{0}^{L} \theta(x) \hat{\theta}\left(t, \tau_{1}\right)\left[z\left(x, t, \tau_{1}\right)\right]^{2} \mathrm{~d} x \mathrm{~d} t \leq \mathrm{e}^{-\left(\gamma-c_{4}\right)\left(\tau_{1}-\tau_{0}\right)} \int_{0}^{\infty} \int_{0}^{L} \theta(x) \hat{\theta}\left(t, \tau_{0}\right)\left[z\left(x, t, \tau_{0}\right)\right]^{2} \mathrm{~d} x \mathrm{~d} t \\
& +c_{5} \int_{\tau_{0}}^{\tau_{1}} \mathrm{e}^{-\left(\gamma-c_{4}\right)\left(\tau_{1}-\tau\right)}\left[\frac{1}{\gamma} \int_{0}^{\infty} \int_{0}^{L} \theta(x) \hat{\theta}(t, \tau) G\left(x, v^{\prime}, \beta^{\prime}\right)^{2} \mathrm{~d} x \mathrm{~d} t\right. \\
& +\left(\left[q_{1}\right]^{2}+\left[q_{2}\right]^{2}\right) \int_{0}^{\infty} \hat{\theta}(t, \tau) \mathrm{d} t+\int_{0}^{\infty} \int_{0}^{L} \rho(t, \tau)[z(x, t, \tau)]^{2} \mathrm{~d} x \mathrm{~d} t \\
& \left.+\frac{\hat{\theta}(0, \tau)}{\nu(1-\nu)}\left([\nu]^{2}+\int_{0}^{L}[f]^{2} \mathrm{~d} x\right)+\frac{\hat{\theta}(\infty, \tau)}{\eta(1-\eta)}\left([\eta]^{2}+\left[q_{1}\right]^{2}+\left[q_{2}\right]^{2}+\sup _{x} G\left(x, \underline{v}^{\prime}, \underline{\beta}^{\prime}\right)^{2}\right)\right] \mathrm{d} \tau .
\end{aligned}
$$




\section{Existence OF SOlUtions}

Here we use the results of Theorems 2.2 and 4.6, and the assumption (3.14), (3.15), to construct nonstationary solutions of (1.1), (1.2). We emphasize the constructive feature of the algorithm, distinguishing necessary conditions on the parameters from sufficient conditions used above to show that such solutions can be constructed.

The essential features of the system (1.1), (1.2) as a model of two-phase flow are contained in the balance term $F$. Here we shall assume $F$ satisfying only (2.8), (2.24) and the mild regularity assumptions of Theorem 2.2 . Having chosen values

$$
q_{1}>K, q_{2}<0
$$

we have the corresponding stationary solution $\underline{z}$ uniquely determined by appeal to Theorem 2.2 .

Next we determine $\theta$ from (4.13). With both $\underline{z}$ and $\theta$ known, we can check directly that (4.21) holds and in addition that

$$
\frac{1}{4} \theta^{2}\left(\frac{\underline{v}^{2} b_{\beta \beta}(\underline{\beta})}{\theta}\right)_{x}+\theta F_{\beta}(\cdot, \underline{v}, \underline{\beta}) \leq-c<0
$$

holds, with some constant $c$, throughout the interval $(0, L)$.

If so, then Lemma 4.3 holds, with a constant $\gamma$ which can be determined from (4.17). In this case the values of $q_{1}$ and $q_{2}$ are acceptable. (Otherwise, $q_{1}-K$ and/or $\left|q_{2}\right|$ must be reduced.)

The restriction to solutions close to a stationary solution derives from the need to control the higher order terms in (4.14). In particular, there is an $\varepsilon>0$ such that (4.14) implies the stronger condition (4.40) within any open subset of $\Omega$ where

$$
|z-\underline{z}|,\left|z_{x}-\underline{z}_{x}\right|,\left|z^{\prime}-\underline{z}\right| \leq \varepsilon
$$

We observe that lemmas 4.2, 4.4, 4.5 and (4.40) suffice to establish (4.41). Therefore in order to continue solutions of (3.3), satisfying (4.41), it will suffice to show that (3.13) remains satisfied.

With this value of $\varepsilon$ in (3.13), let $\delta$ be the corresponding value obtained from (3.14). If (3.14) is satisfied at $\tau=0$, then solutions $z$ and $z^{\prime}$ exist, and satisfy (4.41), for values of $\tau$ sufficiently small that (3.14) remains satisfied by both $z$ and $z^{\prime}$. In general, there is also a restriction on the adopted function $\hat{\theta}$, namely that

$$
\operatorname{supp} \hat{\theta} \subseteq\left\{\mathrm{t} \leq \mathrm{c}_{2} \tau-\mathrm{c}_{3}\right\}
$$

In (5.4), if $\underline{z}=\underline{z}^{\prime}$ the constants $c_{2}, c_{3}$ may correspond either to $z$ or $z^{\prime}$, as one may interchange $z$ with $z^{\prime}$ in $(3.14)$.

Now in principle (4.41) can be used to show that (3.14) is satisfied for all $\tau$, so that the solutions continue indefinitely, satisfying (4.41).

For given "data" of the form $F, q_{1}, q_{2}, \nu, f$, our first application of this strategy corresponds to the choice

$$
z^{\prime}(x, t, \tau)=\underline{z}(x)
$$

the corresponding stationary solution. This choice of $z^{\prime}$ obviously satisfies (3.14) for all $\tau$ and is smooth, so in this case no restriction of the form (5.4) on $\hat{\theta}$ is needed.

Next we choose "numerical" parameters $\eta, c_{4}$, satisfying

$$
0<\eta<1,0<c_{4}<\gamma
$$

and set

From (5.5) and (3.7),

$$
\hat{\theta}(t, \tau)=\mathrm{e}^{c_{4} t}
$$

$$
f^{\prime}(x)=\nu \underline{\beta}(x)+(1-\nu) \underline{v}(x), \quad 0<x<L .
$$


We need initial data of the form $z(\cdot, \cdot, 0)$ for the system $(3.3)$, which is assumed to satisfy

$$
\begin{gathered}
f(x)=\nu \beta(x, 0,0)+(1-\nu) v(x, 0,0), \\
\sup _{\substack{0<x<L \\
t \geq 0}}\left(|z(x, t, 0)-\underline{z}(x)|+\left|z_{x}(x, t, 0)-\underline{z}_{x}(x)\right|+\left|z_{t}(x, t, 0)\right|\right) \leq \delta / 3, \\
\int_{0}^{\infty} \int_{0}^{L} \theta(x) \mathrm{e}^{c_{4} t}|z(x, t, 0)-\underline{z}(x)|^{2} \mathrm{~d} x \mathrm{~d} t \leq \delta / 3, \\
\frac{c_{5}}{\nu(1-\nu)\left(\gamma-c_{4}\right)} \int_{0}^{L}\left(f(x)-f^{\prime}(x)\right)^{2} \mathrm{~d} x \leq \delta / 3,
\end{gathered}
$$

where $c_{5}$ is the constant in (4.41).

We have thus specified an initial/boundary value problem for the system (3.3), (2.12), (2.13), (3.7), (3.8). Denote by $z$ an entropy weak solution thereof, for as long as it exists. In practice, the constant $\delta$ is not knowable; if the conditions of Theorem 4.6 are satisfied, one knows that a sufficiently small positive $\delta$ exists so that a solution will be obtained. In practice, the conditions (5.10)-(5.12) are uncertain. Indeed, if $f-f^{\prime}$ is too large, then (5.12) cannot be satisfied by any choice of $z(\cdot, \cdot, 0)$. So one chooses $z(\cdot, \cdot, 0)$ smooth, satisfying (5.9), (3.9), (3.10) and such that the integral in (5.11) is finite, and runs the computation. If the solution blows up, either $f-f^{\prime}$ is too large or else the choice of $z(\cdot, \cdot, 0)$ needs modification. Hereafter, of course, we assume that the conditions $(5.10)-(5.12)$ were satisfied.

From (5.10), (5.11), it is clear that the solution $z$ satisfies (3.14) for sufficiently small positive $\tau$. But for any $\tau$ such that (3.14) holds for $z$, we can apply (4.41) with $z^{\prime}$ given in (5.5).

Using (5.11), (5.12) in (4.41) and setting $\tau_{0}=0$ gives

$$
\int_{0}^{\infty} \int_{0}^{L}\left|z\left(x, t, \tau_{1}\right)-\underline{z}(x)\right|^{2} \mathrm{e}^{c_{4} t} \mathrm{~d} x \mathrm{~d} t \leq \frac{\delta}{3},
$$

so the solution $z$ can be continued indefinitely in $\tau$, satisfying (3.14) and (5.13).

We employ a similar strategy to show that $z(\cdot, \cdot, \tau)$ converges to a limit as $\tau \rightarrow \infty$. Let $c_{2}, c_{3}$, be the constants obtained in (3.15) corresponding to the solution $z$; without loss of generality we assume $c_{3} \geq 0$.

We choose $\hat{\theta}$ satisfying (5.4), of the form

$$
\hat{\theta}(t, \tau)=\left\{\begin{array}{l}
1, \quad t \leq c_{2} \tau-c_{3}-1 \\
c_{2} \tau-c_{3}-t, c_{2} \tau-c_{3}-1<t<c_{2} \tau-c_{3} \\
0, \quad t \geq c_{2} \tau-c_{3}
\end{array}\right.
$$

and for this application

$$
z^{\prime}(x, t, \tau)=z(x, t, \tau+T)
$$

for some $T>0$.

For $\hat{\theta}$ as given in (5.14), we have (4.39) holding with

$$
\rho(t, \tau)= \begin{cases}1, & c_{2} \tau-c_{3}-1<t<c_{2} \tau-c_{3} \\ 0, & \text { otherwise. }\end{cases}
$$


With $\tau_{0}=0, \tau_{1}$ large in (4.41), using (5.14), (5.15), (5.16) we have

$$
\begin{aligned}
\int_{0}^{\infty} \int_{0}^{L} \theta(x) \hat{\theta}(t, \tau) \mid z(x, t, & \left.\tau_{1}\right)-\left.z^{\prime}\left(x, t, \tau_{1}\right)\right|^{2} \mathrm{~d} x \mathrm{~d} t \\
& \leq c_{5} \int_{c_{2} \tau_{1}-c_{3}-1}^{c_{2} \tau_{1}-c_{3}} \int_{0}^{L} \theta(x)\left|z\left(x, t, \tau_{1}\right)-z^{\prime}(x, t, \tau)\right|^{2} \mathrm{~d} x \mathrm{~d} t \\
& \leq c_{5} \mathrm{e}^{-c_{4}\left(c_{2} \tau_{1}-c_{3}-1\right)} \int_{c_{2} \tau_{1}-c_{3}}^{c_{2} \tau_{1}-c_{3}-1} \mathrm{e}^{c_{4} t} \int_{0}^{L} \theta(x)\left|z\left(x, t, \tau_{1}\right)-z^{\prime}\left(x, t, \tau_{1}\right)\right|^{2} \mathrm{~d} x \mathrm{~d} t \\
& \leq c \mathrm{e}^{-c_{4} c_{2} \tau_{1}} \int_{0}^{\infty} \int_{0}^{L} \mathrm{e}^{c_{4} t}\left(\left|z\left(x, t, \tau_{1}\right)-\underline{z}(x)\right|^{2}+\left|z\left(x, t, \tau_{1}+T\right)-\underline{z}(x)\right|^{2}\right) \theta(x) \mathrm{d} x \mathrm{~d} t \\
& \leq c \delta \mathrm{e}^{-c_{4} c_{2} \tau_{1}}
\end{aligned}
$$

using (5.13) and (5.15) in the last step.

In (5.17), the constant $c_{4}>0$ is the constant chosen in (5.6), as appearing in (5.13), so the right side of (5.17) vanishes in the limit $\tau_{1} \rightarrow \infty$. As $T$ is arbitrary, (5.17) suffices to establish the existence of $\bar{z}=\bar{z}(x, t)$ such that

$$
\int_{0}^{\infty} \int_{0}^{L} \theta(x) \mathrm{e}^{c_{4} t}|z(x, t, \tau)-\bar{z}(x, t)|^{2} \mathrm{~d} x \mathrm{~d} t \rightarrow 0 \text { as } \tau \rightarrow \infty .
$$

As $z$ satisfies (3.3), from (5.18) and (3.10) it follows that $\bar{z}$ is an entropy weak solution of (3.3). As $\bar{z}$ is independent of $\tau$, it also satisfies (1.1), (1.2) weakly. But from (5.18) and (3.13), $\left|\bar{z}_{x}\right|$ is bounded; then from (1.1), (1.2), it follows that $\left|\bar{z}_{t}\right|$ is bounded. Now again using (5.18), we have $\bar{z}$ satisfying (3.9), (3.10), (3.7) and

$$
\bar{z}(x, t) \rightarrow \underline{z}(x) \text { as } t \rightarrow \infty .
$$

From (5.19), it follows that $\bar{z}$ satisfies (3.8) for any choice of $\eta$.

For fixed parameters $F, q_{1}, q_{2}, \nu, f$, the solution $\bar{z}$ is unique, by appeal to (4.41). Thus $\bar{z}$ is independent of the "numerical" parameters $\eta, c_{4}$, and $z(\cdot, \cdot, 0)$. We summarize these results as follows:

Theorem 5.1. Let $q_{1}, q_{2}, \nu, f, F$ be such that (5.1), (4.21), (5.2), and (5.12) hold, with $f^{\prime}$ obtained from (5.8) and some $c_{4}$. Assume $f-f^{\prime}$ sufficiently small that (5.9)-(5.12), (3.9), (3.10) can be satisfied by some $z(\cdot, \cdot, 0)$ for which the assumption (3.14), (3.15) is valid. Assume F satisfying the assumptions of Theorem 2.2.

Then there exists a unique solution of (1.1), (1.2), (3.9), (3.10), (3.7), (5.19), in the class of functions with uniformly bounded first partial $x-$ and $t$ - derivatives.

A semigroup property follows immediately from the uniqueness of the solution functions so constructed.

Corollary 5.2. For a given solution $\bar{z}$, let $\tilde{z}$ be the solution of (1.1), (1.2), (3.9), (3.10), (5.19), and (3.7) with a function

$$
\tilde{f}(x)=\nu \bar{\beta}(x, T)+(1-\nu) \bar{v}(x, T)
$$

for some $T>0$. Then

$$
\tilde{z}(x, t)=z(x, t+T)
$$

for all $x \in[0, L], t>0$. 
Finally we use (4.41) to compare a solution $\bar{z}$ corresponding to parameters $q_{1}, q_{2}, \nu, f, F$ with another solution $\bar{z}^{\prime}$ corresponding to $q_{1}^{\prime}, q_{2}^{\prime}, \nu^{\prime}, f^{\prime}, F^{\prime}$ of the form (2.19). Since $\left|\bar{z}_{k}\right|$ (or $\left|\bar{z}_{x}^{\prime}\right|$ ) is bounded, no restriction of the form (5.4) on the choice of $\hat{\theta}$ is needed. However, in general the corresponding stationary solutions are different, and existence of the integrals in (4.41) depends on the choice of $\hat{\theta}$.

Thus we take

$$
\hat{\theta}(t, \tau)=\mathrm{e}^{-c_{4} t}, \quad c_{4} \in(0, \gamma)
$$

satisfying (4.39) with $\rho=0$, and $\tau_{1}-\tau_{0}=1$. In (5.22) and below, $\gamma$ may correspond to either $\bar{z}$ or $\bar{z}^{\prime}$. The following results from (4.41).

Theorem 5.3. Let $\bar{z}, \bar{z}^{\prime}$ be two solutions obtained in theorem 5.1, corresponding to different parameters. Then for any $c_{4} \in(0, \gamma)$,

$$
\begin{aligned}
\int_{0}^{\infty} \int_{0}^{L} \theta(x) \mathrm{e}^{-c_{4} t}\left|\bar{z}(x, t)-\bar{z}^{\prime}(x, t)\right|^{2} \mathrm{~d} x \mathrm{~d} t \leq \frac{c}{\gamma-c_{4}}\left[\frac{1}{\gamma} \int_{0}^{\infty} \int_{0}^{L} \theta(x) \mathrm{e}^{-c_{4} t} G\left(x, \bar{v}^{\prime}, \bar{\beta}^{\prime}\right)^{2} \mathrm{~d} x \mathrm{~d} t\right. \\
\left.+\frac{\left(q_{1}-q_{1}^{\prime}\right)^{2}+\left(q_{2}-q_{2}^{\prime}\right)^{2}}{c_{4}}+\frac{1}{\nu(\nu-1)}\left(\left(\nu-\nu^{\prime}\right)^{2}+\int_{0}^{L}\left(f(x)-f^{\prime}(x)\right)^{2} \mathrm{~d} x\right)\right] .
\end{aligned}
$$

In the special case $G=0, q_{1}=q_{1}^{\prime}, q_{2}=q_{2}^{\prime}$, it follows that the stationary solutions coincide, and one may pass to the limit $c_{4}=0$ in $(5.23)$.

\section{6. "AVERAGE" SOLUTIONS AND MULTIPLE-VALUED SOLUTIONS}

Appraisal of the system (1.1), (1.2) as a model of incompressible two-phase flow obviously requires the identification of a sufficiently rich set of solutions for comparison of computed solutions with experiment. Thus it is important to maximize the degree of freedom in the choice of problem parameters-restrictions in the choices of these parameters undermine the value of the system as such a model. Restrictions on the form of the obtained solutions, of course, imply corresponding restrictions on the associated problem parameters.

The set of solutions constructed above suffers from several such restrictions. Perhaps the most obvious is the analysis in the small, limited to solutions achieving values close to stationary solutions. This is unavoidable in the above, because of the need to control the higher order terms in (4.14), so as to obtain (4.40). In practice, this restriction may be more apparent than real, depending on the balance term.

As against this, two features of the solutions obtained above are perhaps unacceptable in this context. One is the condition at $t=0$ satisfied by a solution $\bar{z}$. Presumably one wishes to specify a vector quantity

$$
z(x, 0)=\left(\begin{array}{c}
v(x, 0) \\
\beta(x, 0)
\end{array}\right), \quad 0<x<L .
$$

In the above construction, we choose $\nu \in(0,1)$ and compute

$$
f^{\nu}(x)=\nu \beta(x, 0)+(1-\nu) v(x, 0), \quad 0<x<L .
$$

We compute a solution $\bar{z}$ satisfying (3.7) with $f=f^{\nu}$, which thus satisfies

$$
f^{\nu}(x)=\nu \bar{\beta}(x, 0+)+(1-\nu) \bar{v}(x, 0+) .
$$

From (6.2), (6.3), we have

$$
\nu(\beta(\cdot, 0)-\bar{\beta}(\cdot, 0+))+(1-\nu)(v(\cdot, 0)-\bar{v}(\cdot, 0+1)=0,
$$


but in general

$$
z(\cdot, 0) \neq \bar{z}(\cdot, 0+)
$$

A second, related problem arises in the permitted values of $q_{1}, q_{2}$. In a realistic model, these should be permitted to depend on time. The algorithm described above can easily be extended to permit this, say by assuming that $q_{1}, q_{2}$ approach limits as $t \rightarrow \infty$ and computing the stationary solution $\underline{z}$ with these limiting values. However a causality problem results; changes in $q_{1}, q_{2}$ at a given time in general will be reflected in the solution $\mathrm{z}$ at earlier times.

Assuming $q_{1}, q_{2}$ piecewise constant functions of time, the causality problem can be avoided by computing the solution recursively in each interval $\left(t_{j}, t_{j+1}\right), j=0,1, \ldots$, within which $q_{1}$ and $q_{2}$ are constant. For each such interval, we use (3.7) with $f$ given by (6.2) at $t=0$ and given by

$$
f(x)=\nu \bar{\beta}\left(x, t_{j}-0\right)+(1-\nu) \bar{v}\left(x, t_{j}-0\right), \quad 0<x<L,
$$

at each $t_{j}>0$. For each such time interval we use (3.8) with the stationary solution computed using the values of $q_{1}, q_{2}$ at $t=t_{j}+0$.

Denoting by $\bar{z}_{j}, j=0,1, \ldots$, the solutions thus obtained, we identify the corresponding solution by

$$
\bar{z}(\cdot, t)=\bar{z}_{j}\left(\cdot, t-t_{j}\right), \quad t_{j} \leq t<t_{j+1} .
$$

From (6.5), however, the function $\bar{z}$ obtained from (6.7) will have jump discontinuities at each $t_{j}, j=1,2, \ldots$, and in general will not be even a weak solution of (1.1), (1.2).

These problems derive from the lack of hyperbolicity of the system (1.1), (1.2) [10]. As an alternative to adding dissipation terms, thus making the system parabolic, we propose weakening the form of an admissible solution to allow multiple-valued solutions and certain related "average" solutions.

Given data of the form (6.1) at $t=0$, we compute $f^{\nu}$ from (6.2) and a corresponding solution $z(\cdot, \cdot, \nu)$ satisfying (1.1), (1.2), (3.8), (6.3), (3.9), (3.10) for each $\nu \in(0,1)$. Thus we obtain a multiple-valued "solution" achieving a set of values

$$
\{\bar{z}(x, t ; \nu) \mid 0<\nu<1\}
$$

at a given point $(x, t)$.

Collective properties of these sets are described by introducing "average" solutions of the form

$$
\begin{aligned}
Z(x, t) & =\left(\begin{array}{l}
V(x, t) \\
B(x, t)
\end{array}\right) \\
& =\int_{0}^{1}\left(\begin{array}{c}
\mu(1-\nu) \\
\mu(\nu)
\end{array}\right) h(x, t ; \nu) \mathrm{d} \nu, \quad 0 \leq x \leq L, t>0,
\end{aligned}
$$

with

$$
h(x, t ; \nu)=\nu \bar{\beta}(x, t ; \nu)+(1-\nu) \bar{v}(x, t ; \nu)
$$

and $\mu$ a measure on the interval $(0,1)$.

Substituting (6.9) in (6.8) we obtain

$$
\begin{gathered}
V(x, t)=\int_{0}^{1} \mu(1-\nu)(\nu \bar{\beta}(x, t ; \nu)+(1-\nu) \bar{v}(x, t ; \nu)) \mathrm{d} \nu, \\
B(x, t)=\int_{0}^{1} \mu(\nu)(\nu \bar{\beta}(x, t ; \nu)+(1-\nu) \bar{v}(x, t ; \nu)) \mathrm{d} \nu .
\end{gathered}
$$


In general each of the $z(\cdot, 0 ; \nu)$ satisfies (6.5). However, simple conditions on the measure $\mu$ assure that the average $Z$ satisfies the desired conditions at $t=0$ and at $t=\infty$.

Theorem 6.1. Assume that $\mu$ satisfies

$$
\int_{0}^{1} \mu(\nu) \mathrm{d} \nu=1
$$

and

$$
\int_{0}^{1} \nu \mu(\nu) \mathrm{d} \nu=1
$$

then

$$
Z(\cdot, 0+)=z(\cdot, 0) \text {. }
$$

Proof. We use (6.4) in each of (6.10) and (6.11), simplifying by use of (6.12), (6.13), thus obtaining

$$
\begin{aligned}
V(x, 0+) & =\int_{0}^{1} \mu(1-\nu)(\nu \beta(x, 0)+(1-\nu) v(x, 0)) \mathrm{d} \nu \\
& =v(x, 0),
\end{aligned}
$$

and

$$
\begin{aligned}
B(x, 0+) & =\int_{0}^{1} \mu(\nu)(\nu \beta(x, 0)+(1-\nu) v(x, 0) \mathrm{d} \nu \\
& =\beta(x, 0) .
\end{aligned}
$$

Hereafter we assume (6.12), (6.13) hold and in addition that

$$
\operatorname{supp} \mu \subseteq[\mathrm{a}, 1-\mathrm{a}] \text { for some } \mathrm{a} \in\left(0, \frac{1}{2}\right)
$$

Next we show that as $\bar{z}(\cdot, \cdot ; \nu)$ becomes independent of $\nu$ at some point it becomes equal to the average $Z$ at that point.

Theorem 6.2. Let $(x, t)$ be a point at which

$$
|\bar{z}(x, t ; \nu)-\tilde{z}(x, t)| \leq \varepsilon, \quad a \leq \nu \leq 1-a
$$

for some $\tilde{z}(z, t)$ and some $\varepsilon \geq 0$; then

$$
|Z(x, t)-\tilde{z}(x, t)| \leq c \varepsilon .
$$

Proof. This is immediate from (6.10), (6.11), using (6.12), (6.13), and (6.18). The constant $c$ in (6.19) depends only on $\int_{0}^{1}|\mu(\nu)| \mathrm{d} \nu$.

The assumption (6.17) is needed for a corollary.

\section{Corollary.}

$$
Z(\cdot, t) \rightarrow \underline{z} \text { as } \mathrm{t} \rightarrow \infty
$$

Proof. By appeal to theorem 5.3, the condition (5.19) is uniform in $\nu$ within the interval $[a, 1-a]$. For $t$ large, (6.18) holds with $\tilde{z}(\cdot, t)=\underline{z}$ and $\varepsilon$ small, and (6.19) follows. 
Averaged quantities $Z$ obtained from (6.8), (6.9) in general do not satisfy the nonlinear system (1.1), (1.2) or the nonlinear boundary conditions (3.9), (3.10). However, they do satisfy an approximate weak form of the corresponding linearized system, with one additional assumption on $\mu$.

Lemma 6.3. Assume $\mu$ satisfying

$$
\nu \mu(\nu)=(1-\nu) \mu(1-\nu), \quad a \leq \nu<\frac{1}{2}
$$

then for any $p, r \in \mathbb{R}$

$$
p B+r V=\int_{0}^{1} \nu \mu(\nu)(p \bar{\beta}(\cdot, \cdot ; \nu)+r \bar{v}(\cdot, \cdot ; \nu)) \mathrm{d} \nu+\int_{0}^{1}(1-\nu) \mu(\nu)(p \bar{v}(\cdot, \cdot ; \nu)+r \bar{\beta}(\cdot, \cdot ; 1-\nu)) \mathrm{d} \nu .
$$

Remark. It is not difficult to construct $\mu(\nu)$ satisfying (6.12), (6.13), (6.17) and (6.21). An example with support at only three points, and thus attractive for computations, is given by

$$
\mu(\nu)=-3 \delta\left(\nu-\frac{1}{4}\right)+5 \delta\left(\nu-\frac{1}{2}\right)-\delta\left(\nu-\frac{3}{4}\right) .
$$

From (6.12), (6.13), the measure $\mu$ cannot be nonnegative valued.

At a point where theorem 6.2 holds with $\varepsilon$ small, the second right-hand term in $(6.22)$ is $O(\varepsilon)$.

Proof. We simply use (6.10), (6.11) in the left side of (6.22) and simplify using (6.21).

Theorem 6.4. Let $\Phi, \Psi$ be smooth functions of $x, t$ of compact support within $[0, L] \times(0, \infty)$ and satisfying

$$
\operatorname{supp} \Phi \cap\{\mathrm{x}=0\}=\emptyset
$$

and

$$
\operatorname{supp} \Psi \cap\{\mathrm{x}=\mathrm{L}\}=\emptyset
$$

then $Z$ satisfies

$$
\begin{aligned}
\int_{0}^{\infty} \int_{0}^{L}[ & \Phi_{t}(x, t) B(x, t)+\Phi_{x}(x, t)\left(\left(K-\underline{v}(x) b_{\beta}(\underline{\beta}(x))\right)(B(x, t)-\underline{\beta}(x))\right. \\
& -b(\underline{\beta}(x))(V(x, t)-\underline{v}(x)))] \mathrm{d} x \mathrm{~d} t \\
= & -\int_{0}^{\infty} \int_{0}^{L} \int_{0}^{1}(1-\nu) \mu(\nu)\left[\left(\Phi_{t}(x, t)+\Phi_{x}(x, t)\left(K-\underline{v}(x) b_{\beta}(\underline{\beta}(x))\right) \bar{v}(x, t ; \nu)\right.\right. \\
& \left.-\Phi_{x}(x, t) b(\underline{\beta}(x)) \bar{\beta}(x, t ; 1-\nu)\right] \mathrm{d} \nu \mathrm{d} x \mathrm{~d} t \\
& +O\left(\int_{0}^{\infty} \int_{0}^{L} \int_{0}^{1}\left|\Phi_{x}(x, t)\right||\bar{z}(x, t ; \nu)-\underline{z}(x)|^{2}\right) \mathrm{d} \nu \mathrm{d} x \mathrm{~d} t
\end{aligned}
$$


and

$$
\begin{aligned}
\int_{0}^{\infty} \int_{0}^{L}[ & \bar{\Psi}_{t}(x, t) V(x, t)+\left[\bar{\Psi}_{x}(x, t)\left(K-\underline{v}(x) b_{\beta}(\underline{\beta}(x))+\bar{\Psi}(x, t) F_{v}(x, \underline{v}(x), \underline{\beta}(\nu))\right](V(x, t)-\underline{v}(x))\right. \\
+ & {\left.\left[-\frac{1}{2} \bar{\Psi}_{x}(x, t) \underline{v}(x)^{2} b_{\beta}(\underline{\beta}(x))+\bar{\Psi}(x, t) F_{\beta}(x, \underline{v}(x), \underline{\beta}(x))\right](B(x, t)-\underline{\beta}(x))\right] \mathrm{d} x \mathrm{~d} t } \\
= & -\int_{0}^{\infty} \int_{0}^{L} \int_{0}^{1}(1-\nu) \mu(\nu)\left[\left(-\frac{1}{2} \bar{\Psi}_{x}(x, t) \underline{v}(x)^{2} b_{\beta \beta}(\underline{\beta}(x))+\bar{\Psi}(x, t) F_{\beta}(x, \underline{v}(x), \underline{\beta}(x)) \bar{v}(x, t ; \nu)\right.\right. \\
+ & \left.\left(\bar{\Psi}_{t}(x, t)+\bar{\Psi}_{x}(x, t)\left(K-\underline{v}(x) b_{\beta}(\underline{\beta}(x))\right)+\bar{\Psi}(x, t) F_{v}(x, \underline{v}(x), \underline{\beta}(x))\right) \bar{\beta}(x, t ; 1-\nu)\right] \mathrm{d} \nu \mathrm{d} x \mathrm{~d} t \\
& +O\left(\int_{0}^{\infty} \int_{0}^{L} \int_{0}^{1}\left(|\bar{\Psi}(x, t)|+\left|\bar{\Psi}_{x}(x, t)\right|\right)|\bar{z}(x, t ; \nu)-\underline{z}(x)|^{2} \mathrm{~d} \nu \mathrm{d} x \mathrm{~d} t\right) .
\end{aligned}
$$

Proof. Throughout we use the abbreviations $\bar{z}=\bar{z}(x, t ; \nu), \Phi=\Phi(x, t), \bar{\Psi}=\bar{\Psi}(x, t), \underline{z}=\underline{z}(x)$.

We introduce a weak form of (1.1), (3.9) satisfied by $\bar{z}$ for all $\nu$. Since $\underline{z}$ satisfies $(2.9)$ and (2.12), partial integrations and (6.24) give

$$
\begin{aligned}
0 & =-\int_{0}^{\infty} \int_{0}^{L} \Phi\left(\bar{\beta}_{t}+(K \bar{\beta}-\bar{v} b(\bar{\beta}))_{x}\right) \mathrm{d} x \mathrm{~d} t \\
& =q_{1} \int_{0}^{\infty} \Phi(L, t) \mathrm{d} t+\int_{0}^{\infty} \int_{0}^{L}\left[\Phi_{t} \bar{\beta}+\Phi_{x}(K \bar{\beta}-\bar{v} b(\bar{\beta}))\right] \mathrm{d} x \mathrm{~d} t \\
& =\int_{0}^{\infty} \int_{0}^{L}\left[\Phi_{t} \bar{\beta}+\Phi_{x}(K \bar{\beta}-\bar{v} b(\bar{\beta})-K \underline{\beta}+\underline{v} b(\underline{\beta}))\right] \mathrm{d} x \mathrm{~d} t \\
& =\int_{0}^{\infty} \int_{0}^{L}\left[\Phi_{t} \bar{\beta}+\Phi_{x}\left(K-\underline{v} b_{\beta}(\underline{\beta})\right)(\bar{\beta}-\underline{\beta})-\Phi_{x} b(\underline{\beta})(\bar{v}-\underline{v})+O\left(\left|\Phi_{x}\right||\bar{z}-\underline{z}|^{2}\right)\right] \mathrm{d} x \mathrm{~d} t .
\end{aligned}
$$

Multiplying (6.28) by $\nu \mu(\nu)$ and integrating with respect to $\nu$, using (6.24) with

$$
p=\Phi_{t}+\Phi_{x}\left(K-\underline{v} b_{\beta}(\underline{\beta})\right), \quad r=-\Phi_{x} b(\underline{\beta})
$$

we obtain (6.26). 
We obtain (6.27) similarly. A weak form of (1.2), (3.10), using (2.10), (2.13) and (6.25), is

$$
\begin{aligned}
0= & -\int_{0}^{\infty} \int_{0}^{L} \bar{\Psi}\left[\bar{v}_{t}+\left(K \bar{v}-\frac{1}{2} \bar{v}^{2} b_{\beta}(\bar{\beta})\right)_{x}-F(x, \bar{v}, \bar{\beta})\right] \mathrm{d} x \mathrm{~d} t \\
= & -q_{2} \int_{0}^{\infty} \bar{\Psi}(0, t) \mathrm{d} t+\int_{0}^{\infty} \int_{0}^{L}\left[\bar{\Psi}_{t} \bar{v}+\bar{\Psi}_{x}\left(K \bar{v}-\frac{1}{2} \bar{v}^{2} b_{\beta}(\bar{\beta})\right)+\bar{\Psi} F(x, \bar{v}, \bar{\beta})\right] \mathrm{d} x \mathrm{~d} t \\
= & \int_{0}^{\infty} \int_{0}^{L}\left[\bar{\Psi}_{t} \bar{v}+\bar{\Psi}_{x}\left(K \bar{v}-\frac{1}{2} \bar{v}^{2} b_{\beta}(\bar{\beta})-K \underline{v}+\frac{1}{2} \underline{v}^{2} b_{\beta}(\underline{\beta})\right)+\bar{\Psi}(F(x, \bar{v}, \bar{\beta})-F(x, \underline{v}, \underline{\beta}))\right] \mathrm{d} x \mathrm{~d} t \\
= & \int_{0}^{\infty} \int_{0}^{L}\left[\bar{\Psi}_{t} \bar{v}+\left(\bar{\Psi}_{x}\left(K-\underline{v} b_{\beta}(\underline{\beta})\right)+\bar{\Psi} F_{v}(x, \underline{v}, \underline{\beta})\right)(\bar{v}-\underline{v})\right. \\
& +\left(-\frac{1}{2} \bar{\Psi}_{x} \underline{v}^{2} b_{\beta \beta}(\underline{\beta})+\bar{\Psi} F_{\beta}(x, \underline{v}, \underline{\beta})\right)(\bar{\beta}-\underline{\beta})+O\left(\left(|\bar{\Psi}|+\left|\bar{\Psi}_{x}\right|\right)|\bar{z}-\underline{z}|^{2}\right] \mathrm{d} x \mathrm{~d} t .
\end{aligned}
$$

Multiplying (6.30) by $\nu \mu(\nu)$ and integrating, using (6.22) with

we obtain (6.27).

$$
p=-\frac{1}{2} \bar{\Psi}_{x} \underline{v}^{2} b_{\beta \beta}(\underline{\beta})+\bar{\Psi} F_{\beta}(\cdot, \underline{v}, \underline{\beta}), \quad r=\bar{\Psi}_{t}+\bar{\Psi}_{x}\left(K-\underline{v} b_{\beta}(\underline{\beta})\right)+\bar{\Psi} F_{v}(\cdot, \underline{v}, \underline{\beta}),
$$

In describing incompressible two-phase flow, the average $Z$ is superior to an individual solution $z(\cdot, \cdot ; \nu)$ in some respects and inferior in others. The average $Z$ satisfies both components of the condition (6.1) at $t=0$, and is continuous in $t$ when $q_{1}, q_{2}$ are given as piecewise constant functions of time and the solution is computed recursively as described in (6.6), (6.7). As against this, $Z$ satisfies only the approximate equations (6.26), (6.27).

While $Z$ depends on the specific choice of the "numerical parameter" $\mu(\nu)$, the function $z(\cdot, \cdot ; \nu)$ depends on the numerical parameter $\nu$. In the exceptional case in which the data is such that (6.18) applies for all $x, t$ with some small $\varepsilon$, the first right-hand terms of (6.26) and (6.27) are $O(\varepsilon)$. In this case, $Z$ satisfies (1.1), (1.2), (3.9), $(3.10)$ to $O(\varepsilon)$.

\section{REFERENCES}

[1] D. Amadori, L. Gosse and G. Guerra, Global BV entropy solutions and uniqueness for hyperbolic systems of balance laws. Arch. Ration. Mech. Anal. 162 (2002) 327-366.

[2] A. Bressan, Hyperbolic Systems of Conservation Laws: the One-dimensional Cauchy Problem. Oxford University Press (2000).

[3] R.J. DiPerna, Uniqueness of solutions to hyperbolic conservation laws. Indiana Univ. Math. J. 28 (1979) 202-212.

[4] T.N. Dinh, R.R. Nourgaliev and T.G. Theofanous, Understanding the ill-posed two-fluid model, in Proc. of the 10th International Topical Meeting on Nuclear Reactor Thermal Hydraulics (NURETH-10) Seoul, Korea (October 2003).

[5] D.A. Drew and S.L. Passman, Theory of Multicomponent Fluids. Springer, New York (1999).

[6] S.K. Godunov, An interesting class of quasilinear systems. Dokl. Akad. Nauk SSR 139 (1961) 521-523.

[7] M. Ishii, Thermo-fluid dynamic theory of two-phase flow. Eyrolles, Paris (1975).

[8] B.L. Keyfitz, R. Sanders and M. Sever, Lack of hyperbolicity in the two-fluid model for two-phase incompressible flow. Discrete Contin. Dynam. Systems, Series B 3 (2003) 541-563.

[9] B.L. Keyfitz, M. Sever and F. Zhang, Viscous singular shock structure for a nonhyperbolic two-fluid model. Nonlinearity $\mathbf{1 7}$ (2004) 1731-1747.

[10] H.-O. Kreiss and J. Ystrom, Parabolic problems which are ill-posed in the zero dissipation limit. Math. Comput. Model. 35 (2002) 1271-1295.

[11] M.S. Mock, Systems of conservation laws of mixed type. J. Diff. Equations 37 (1980) 70-88.

[12] H. Ransom and D.L. Hicks, Hyperbolic two-pressure models for two-phase flow. J. Comput. Phys. 53 (1984) 124-151.

[13] R. Sanders and M. Sever, Computations with singular shocks (2005) (preprint).

[14] S. Sever, A model of discontinuous, incompressible two-phase flow (2005) (preprint).

[15] H.B. Stewart and B. Wendroff, Two-phase flow: models and methods. J. Comput. Phys. 56 (1984) 363-409. 